\title{
Zur Bedeutung des Hippokra- tischen Eides in der heutigen Zeit
}

\author{
Josias Mattlia, Ursula Knirsch ${ }^{b}$, Raimund Klesse ${ }^{c}$, Sabine Vuilleumier-Koch ${ }^{d}$ \\ Vorstandsmitglieder der Hippokratischen Gesellschaft Schweiz \\ ${ }^{a}$ Dr. med., Facharzt für Allgemeine Innere Medizin, Mitglied der FMH, Val Müstair; ${ }^{b}$ Dr. med., Fachärztin für Neurologie, Mitglied der FMH, Zürich; \\ ${ }^{c}$ Dr. med., Facharzt für Psychiatrie und Psychotherapie, Mitglied der FMH, Chur; ${ }^{d}$ Dr. med., Fachärztin für Psychiatrie und Psychotherapie, Mitglied der FMH, \\ Greifensee
}

Die Notwendigkeit eines neuen, verpflichtenden Eides wurde postuliert und andiskutiert [1]. Begründet wird dies mit aktuellen Problemen wie der Ökonomisierung in der Medizin. Gleichzeitig versucht man, die Bedeutung des Hippokratischen Eides zu relativieren und ihn als unzeitgemäss abzutun. Die Hippokratische Gesellschaft Schweiz legt die unveränderte Aktualität des Hippokratischen Eides dar.

\section{Der Hippokratische Eid als «Fels in der Brandung» [2]}

Der Hippokratische Eid stellt seit fast 2500 Jahren eine der wichtigsten ethischen Grundlagen des ärztlichen Handelns dar. Die darin enthaltenen Werte sind zeitunabhängig und haben verschiedenste geschichtliche Epochen überdauert. Der Eid verkörpert damit eine überragende normative Kraft. Er ist religiös unabhängig und beinhaltet die zentralen Grundwerte der Medizin. Jeder Arzt sollte diese Grundlagen kennen, verinnerlichen und danach handeln. Deshalb wollen wir hier Text und Bedeutung dieses hochaktuellen Dokuments in Erinnerung rufen (siehe Kasten).

\section{Der Hippokratische Eid - verlässliche Richtschnur seit 2500 Jahren}

Der Hippokratische Eid ist eine moralische Selbstverpflichtung des Arztes. Der Schwur den Göttern gegenüber zeigt die Ernsthaftigkeit und Unbedingtheit des Verhaltenskodex. Die Verpflichtung basiert ganz auf dem Gedanken des «nihil nocere»: dem Patienten Nutzen bringen und keinen Schaden zufügen. Im Zentrum steht das Tötungsverbot, der unbedingte Schutz des Lebens als Grundlage jeder ärztlichen Tätigkeit. In der Frage der Ehrfurcht vor dem Leben muss der Arzt absolut sicher sein, da sonst der Aufbau eines Vertrauensverhältnisses zwischen Arzt und Patient nicht möglich ist. Auch die klare Verpflichtung zur Wahrung des Arztgeheimnisses ist eine notwendige Grundlage für diese Vertrauensbeziehung.
L'importance actuelle du serment d'Hippocrate Depuis près de 2500 ans, le serment d'Hippocrate est le principal fondement éthique de l'activité médicale qui repose sur le principe du «nihil nocere», autrement dit servir le patient sans lui nuire. L'interdiction de tuer en est la base et le médecin doit placer le respect de la vie au centre de la relation thérapeutique de sorte à pouvoir établir un lien de confiance avec le patient. La relation thérapeutique comprend également l'obligation de garder le secret et le devoir de diligence professionnel. Selon le serment d'Hippocrate, la profession médicale est une vocation au sens d'un engagement de toute une vie qui doit être accompli dans le plus grand respect en assumant la responsabilité la plus élevée. Nous, médecins, devrions continuer à prendre soin et à être les garants de la santé de nos patients en nous fondant sur cette base.

Der Eid verlangt vom Arzt absolute Sorgfalt in der Berufsausübung. Er muss seine Grenzen kennen und darf nicht risikoreich handeln. Dies kommt im Versprechen zum Ausdruck, keinen Blasensteinkranken zu operieren, was damals nicht zur Ausbildung des Arztes gehörte. Der Eid verlangt vom Arzt aber auch eine Vorbildfunktion in Verhalten und eigener Lebensführung. Dazu gehörte bereits damals das Verbot von sexuellen Übergriffen auf die Patienten. Hippokrates misst dem Lernen des Studenten in der persönlichen Beziehung zum ärztlichen Lehrer grösste Bedeutung bei. Den geforderten Respekt und die Achtung dem erfahrenen 


\section{Hippokratischer Eid}

1. Ich schwöre bei Apollon dem Arzt und bei Asklepios, Hygieia und Panakeia sowie unter Anrufung aller Götter und Göttinnen als Zeugen, dass ich nach Kräften und gemäss meinem Urteil diesen Eid und diesen Vertrag erfüllen werde:

2. Denjenigen, der mich diese Kunst gelehrt hat, werde ich meinen Eltern gleichstellen und das Leben mit ihm teilen; falls es nötig ist, werde ich ihn mitversorgen. Seine männlichen Nachkommen werde ich wie meine Brüder achten und sie ohne Honorar und ohne Vertrag diese Kunst lehren, wenn sie sie erlernen wollen. Mit Unterricht, Vorlesungen und allen übrigen Aspekten der Ausbildung werde ich meine eigenen Söhne, die Söhne meines Lehrers und diejenigen Schüler versorgen, die nach ärztlichem Brauch den Vertrag unterschrieben und den Eid abgelegt haben, aber sonst niemanden.

3. Die diätetischen Massnahmen werde ich nach Kräften und gemäss meinem Urteil zum Nutzen der Kranken einsetzen, Schädigung und Unrecht aber ausschliessen.

4. Ich werde niemandem, nicht einmal auf ausdrückliches Verlangen, ein tödliches Medikament geben, und ich werde auch keinen entsprechenden Rat erteilen; ebenso werde ich keiner Frau ein Abtreibungsmittel aushändigen.

5. Lauter und gewissenhaft werde ich mein Leben und meine Kunst bewahren.

6. Auf keinen Fall werde ich Blasensteinkranke operieren, sondern ich werde hier den Handwerkschirurgen Platz machen, die darin erfahren sind.

7. In wie viele Häuser ich auch kommen werde, zum Nutzen der Kranken will ich eintreten und mich von jedem vorsätzlichen Unrecht und jeder anderen Sittenlosigkeit fernhalten, auch von sexuellen Handlungen mit Frauen und Männern, sowohl Freien als auch Sklaven.

8. Über alles, was ich während oder ausserhalb der Behandlung im Leben der Menschen sehe oder höre und das man nicht nach draussen tragen darf, werde ich schweigen und es geheimhalten.

9. Wenn ich diesen meinen Eid erfülle und ihn nicht antaste, rühmt bei allen Menschen für alle Zeiten; wenn ich ihn aber übertrete und meineidig werde, dann soll das Gegenteil davon geschehen.

(Übersetzung von Axel W. Bauer)

1 Dieser Text basiert auf der Grundlage des Vortrages "Überlebt Hippokrates?» von Jakob Gehring und Josias Mattli, gehalten auf dem 1. Symposium Medizin und Ethik, Davos, 1998.

2 (Im Griechischen wird die besondere Bedeutung durch eine vierfache Verneinung hervorgehoben: «Ich werde aber nicht und ganz und gar nicht (und) niemandem als (dazu) Gebetener ein todbringendes Mittel geben, und ich werde auch nicht einen solchen Ratschlag vorzeigen", Übersetzung Gehring und Mattli)

Arzt gegenüber, der ihn die ärztliche Kunst und Ethik lehrt, vergleicht Hippokrates mit der Beziehung zu den Eltern.

Der Eid sieht den Arztberuf als Berufung im Sinne einer Lebensaufgabe, der mit höchstem Respekt und Verantwortungsgefühl zu begegnen ist.

Dieser kurzen Zusammenfassung der grundlegenden Inhalte des Hippokratischen Eides folgt nun eine breitere Darlegung der hippokratischen ärztlichen Haltung, wie sie sich aus der Übersetzung weiterer Schriften des Corpus Hippocraticum ergibt ${ }^{1}$ :

Nach hippokratischer Auffassung begegnet der Arzt seinem Patienten als Mitmensch, der sich ihm in seiner individuellen Ganzheit auf Augenhöhe nähert und mit ihm eine Vertrauensbeziehung aufbaut. Damit ist so möge ich mein Leben und meine Kunst geniessen, ge-

er mehr als nur ein Wissender, der seinen Dienst leistet, indem er die ihm anvertrauten Informationen nutzt, Kenntnisse vermittelt, praktische Kunstgriffe und Techniken anwendet und als Fachmann zur rechten Zeit das Notwendige tut. Der Mensch wird nicht nur als Zusammenwirken von hochspezialisierten Organen betrachtet. Weit darüber hinaus geht die Betrachtungsweise, die ihn als unauflösbare Einheit von Leib, Seele und Geist erfasst. Darum ergreift und betrifft die Krankheit den Menschen nicht nur als körperliches oder psychisches Leiden eines Teilbereichs, sondern immer in der Ganzheit seiner Existenz.

Aus dieser Erkenntnis ergibt sich zwingend: Wer dem Leidenden wirklich helfen möchte, muss ihm ganzheitlich begegnen: seine Persönlichkeit in all ihren Dimensionen erfassen und sich selber in diese Du-IchBeziehung ganz einbringen. Der gute Arzt wirft die eigene Existenz in die Waagschale seines Berufes. Er übt nicht nur eine Funktion aus. Sein Charakter, seine Ehrlichkeit, sein guter Wille und seine Lebensführung werden zu einem heilenden Faktor bei der Gesundung des Patienten.

Von dieser ganzheitlichen Sicht her werden fundamentale Aspekte ärztlichen Verhaltens versteh- und begründbar:

Der Arzt ist dem Leben verpflichtet. Im Kernteil des Hippokratischen Eides wird die Erhaltung des Lebens als wichtigster Grundwert verankert. Im griechischen Originaltext finden wir dazu eine vierfache Verneinung von jeglicher Tötung:

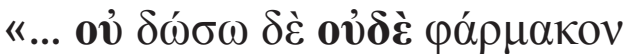

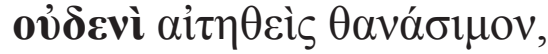

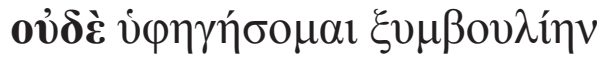 $\tau$ นоฑ์ $\delta \varepsilon \ldots{ }^{2}{ }^{2}$}

Dies bildet die Grundlage des Vertrauens zum Arzt, das darauf aufbaut, dass dieser dem Patienten keinesfalls willentlich schaden werde - das Prinzip des «nihil nocere».

Dass der Arzt dazu seine persönliche Lebensführung vertrauenswürdig gestalte, ohne Ausnahme nur Gutes mit sich bringe, sich auf keinen Fall auf Unrecht einlasse, dass das Gebiet sexueller Begegnung aus seiner Berufstätigkeit ausgeklammert werde, und schliesslich auch das unerbittliche Gebot, über Gespräche am Patientenbett zu schweigen - das alles weist auf das eine Anliegen hin: Das menschliche Umfeld des therapeutischen Dialogs soll vom Grundwert des Vertrauens getragen sein. 
Vertrauen hat mit Hingabe zu tun, mit gutem Willen, mit Verständnis und Hilfsbereitschaft, die der Patient selbstverständlich erwarten darf. Er soll nach Hippokrates wissen: Mein Arzt versteht mich in einem tiefen Sinn, in meinem ganzen Umfeld, und mit ihm begegnet mir nur die gute Absicht. Auch wenn er mir Schmerzen zufügen oder meine Wünsche nicht erfüllen wird - nie werde ich deshalb ihm gegenüber ein Gefühl wie Furcht hegen müssen.

Vergessen wir mit Hippokrates das Eine nicht: Das ungeteilte Vertrauensverhältnis bildet die fundamentale Voraussetzung heilenden Tuns.

Es geht ihm also nicht nur um praktische Normen oder um gesellschaftlich zeitbedingte Konventionen; ärztliche Ethik richtet sich bei Hippokrates nach Grundwerten menschlicher Existenz. Im historischen Kontext wird die Heiligkeit des Lebens verbürgt durch die überzeitliche Kraft der Beziehung zu den Göttern. Albert Schweitzer greift dies später auf im Begriff der «Ehrfurcht vor dem Leben» [3].

In die entgegengesetzte Richtung zeigen die erschreckenden Aspekte der Medizingeschichte des 20. Jahrhunderts: Wo das menschliche Leben - in seiner Ganzheit und als Einzelschicksal verstanden - für den Arzt nicht mehr unantastbar sozusagen axiomatisch gegeben ist, nicht mehr jenseits jeder Diskussion feststeht, sondern den veränderbaren Normen einer beliebigen Moral ausgeliefert wird - dort beginnt unmittelbar das Gebiet des Schuldhaften.

Wo der Arzt der Ehrfurcht vor dem Leben nicht mehr unbedingt verpflichtet ist, wird ihm nämlich eine Kompetenz und Verantwortung zugeschoben, die er nicht tragen kann: Als Begutachter über «lebenswert» oder «lebensunwert», über menschliche Belastbarkeit, menschliches Schicksal, als Richter über Leben und Tod, der sein eigenes Urteil auch noch vollstrecken muss. Und wo Ärzte ohne die unabdingbare Ehrfurcht vor dem Geheimnis des Lebens den Forderungen einer politischen oder gesellschaftlichen Ideologie erlagen, da haben sie immer wieder die Grenze zum Kriminellen überschritten. Als Beispiele seien angeführt: Rassenlehre, Eugenik, Euthanasie, Beurteilung als «lebensunwertes Leben" [4], Genozid durch Sterilisation, Vivisektion, «menschliche Versuchstiere» der Wissenschaft gegen ihren Willen [5].

Der Arzt dagegen, der die Grundwerte seiner Berufung jenseits juristischer Gesetze und wissenschaftlicher Regelungen im Respekt vor der Unantastbarkeit des menschlichen Lebens ernst nimmt, rettet nach Hippokrates nicht nur sein eigenes Leben vor Belastung und Schuld; er wird durch seine Haltung auch für seine Patienten zum Vertrauten - und damit zum Helfer

\section{Rückbesinnung auf die hippokratische Ethik}

Immer wieder in der Geschichte wurden die menschlichen Grundwerte missachtet, wie sie im Naturrecht verankert sind und die Grundlage unserer demokratischen Rechtsstaaten bilden. Während der Weltkriege zeigte sich in grauenhaftem Ausmass, wohin eine Medizin ohne Menschlichkeit führen kann [5]. Heute sind wir mit utilitaristischen Konstrukten der im anglo-amerikanischen Umfeld entwickelten Bioethik konfrontiert. Wieder wird die Unantastbarkeit des menschlichen Lebens in Frage gestellt, wieder wird die Würde des Einzelnen aufgrund fragwürdiger Reflexionen über das angebliche Wohl der Vielen beschädigt, wieder spielen wirtschaftliche und ideologische Aspekte eine grosse Rolle.

Den Vorschlag eines neuen, gar noch standesrechtlich verpflichtenden Eides $[1,6]$, der das Tötungsverbot als Grundlage der medizinischen Ethik nicht enthält und stattdessen das Wirtschaftlichkeitsgebot des KVG durch die Hintertür zu einer ethischen Grundlage der Medizin machen will, können wir nicht unterstützen. Warum soll sich die heutige Ärztegeneration nicht auf die hippokratische Ethik besinnen, die seit über zwei Jahrtausenden das ethische Fundament der Medizin darstellt, und auf dieser Grundlage wieder die Fürsorge- und Garantenpflicht für ihre Patienten übernehmen?

\section{Hinweis}

Die Hippokratische Gesellschaft Schweiz und ihre Anliegen werden zu einem späteren Zeitpunkt in der Schweizerischen Ärztezeitung vorgestellt.

Literatur

1 Giger M. Ein Eid für heutige Ärztinnen und Ärzte. Schweiz Ärztezeitung 2015;96(25):930-4.

2 siehe auch Höffe O. Philosophische Ethik: Fahne im Wind oder Fels in der Brandung. In: Schweiz Ärztezeitung 2010;91 (32):1199-202.

3 Schweitzer A. Die Ehrfurcht vor dem Leben. Verlag C. H. Beck; 2003.

4 Binding K, Hoche A. Die Freigabe der Vernichtung lebensunwerten Lebens. Ihr Mass und ihre Form. Leipzig; 1922.

5 siehe auch: Müller Hill B. Tödliche Wissenschaft. Die Aussonderung von Juden, Zigeunern und Geisteskranken 1933-1945. Reinbek: Rowohlt 1984

6 Wils JP et al. Wir müssen über einen neuen Ärzteeid diskutieren. Schweiz Ärztezeitung 2016;97(10):381-3. 